\title{
Determining parameters in multi-pass turning operations under fuzzy environmental
}

\author{
Ricardo Coelho
}

\author{
Department of Statistics and Applied Mathematics, Federal University of Ceará \\ Av. Mister Hull, s/n, Campus do Pici, Bloco 910, 60.440-900, Fortaleza, CE, Brazil \\ Email: rcoelhos@dema.ufc.br
}

\begin{abstract}
Despite convex mathematical optimization methods be applied to general linear optimization problems it can also be applied in a special class of nonlinear optimization. As ambiguity and vagueness are natural and ever-present in real-life situations requiring solutions, it makes perfect sense to attempt to address them using fuzzy convex optimization. In this work, two fuzzy convex approaches based on two fuzzy linear ones are presented. To illustrate the efficiency of these proposals, a manufacturing process problem is solved.
\end{abstract}

Keywords: Fuzzy logic, Fuzzy optimization, Manufacturing process, Cutting operators

\section{Introduction}

Assembling more products by ensuring their quality with the same amount of feedstock, is one of the goals to maximize the profits in a manufacturing process. In modern manufacturing industry, metal cutting process using computer numerical control (CNC) machining is the proper machine tool for batch production. The machining tasks involve the process plan, tool selection, cutting parameter selection and tool trajectory plan based on the required surface tolerance. Cost consciousness with respect to the metal cutting process is an essential element in efficient manufacturing. So, it is essential to analyze the metal cutting operations to manage at economic conditions.

For the purpose of finding the machining parameters to become the production more efficient, Shin and Joo [8] proposed a mathematical formulation to reach this parameters in multi-pass turning operations. Several optimization techniques to solve this problem can be found in $[1,2,6]$. Nevertheless, in most real practical applications one lacks this kind of exact knowledge, and only approximate, vague and imprecise values are known. Moreover, these imprecise values can be dealt with fuzzy logic. In this case, the concept of fuzzy mathematical programming emerges when it is used.

With this in mind, the goal of this work is to determine machining parameters in multi-pass turning operations under fuzzy environmental by using two fuzzy approaches. These approaches solve convex optimization problems under uncertain order relation of the set of constrains and they have been based on methods developed to solve fuzzy linear optimization problems. The first approach has been extended from the Zimmermann's approach [13], where an aspiration level is defined in order to formulate a classical equivalent optimization problem. The second extended approach has been based on Tanaka's approach [11], where a bound interval is defined to do a tradeoff between the satisfaction level and the violation of the set of constraints. Even though these extended approaches has been developed to solve fuzzy convex optimization problems, the manufacturing process problem illustrates the efficiency of them in the nonlinear optimization context.

This work is organized as follows. Section 2 presents the mathematical formulation of a manufacturing process, which needs to determine the machining parameters in multi-pass turning operations. Section 3 introduces two extending approaches that are based on the linear approaches from Zimmerman [13] and Tanaka [11]. To clarify the above developments, a numerical experiment for different values of the total depth of metal to be removed are analyzed in section 4. Finally, conclusions are presented in Section 5.

\section{Mathematical formulation}

The mathematical formulation of this problem that optimizes the minimum cost of the total production can be defined on the following way

$$
\begin{array}{r}
\min U=A_{2}+A_{1} f_{s}^{\left(\frac{q}{p}-1\right)} d_{s}^{\left(\frac{r}{p}\right)}+\sum_{n o r}^{i=1} A_{2} \\
+A_{1} f_{r i}^{\left(\frac{q}{p}-1\right)} d_{r i}^{\left(\frac{r}{p}\right)}+k_{0} t_{p}
\end{array}
$$

where

$$
\begin{aligned}
& A_{1}=\frac{\pi D L k_{0}}{1000 T_{p}}\left[\frac{T_{s}}{C_{0}}\right]^{\frac{1}{p}}\left[T_{s}+\zeta\left(t_{e}+\frac{k_{t}}{k_{0}}\right)\right] \\
& A_{2}=k_{0}\left(h_{1} L+h_{2}\right) \quad \text { and } \quad T_{p}=\frac{T_{s}}{\zeta} .
\end{aligned}
$$

Subject to 


\begin{tabular}{|c|c|}
\hline $\begin{array}{c}A_{1}, A_{2}, D, \zeta, T_{s} \\
C_{0} \\
d_{s} \\
d_{\min } \\
d_{\max } \\
d_{r i} \\
d_{t} \\
f_{s} \\
f_{r i} \\
f_{\min } \\
f_{\max } \\
F_{\max } \\
h_{1}, h_{2} \\
k_{0} \\
k_{1} \\
k_{t} \\
L \\
n o r \\
p, q, r \\
P_{\max } \\
R \\
R_{\max } \\
R_{r} \\
t_{e} \\
t_{p} \\
T_{p} \\
U \\
\nu_{\min } \\
\nu_{\max } \\
\mu, \nu \\
\eta\end{array}$ & 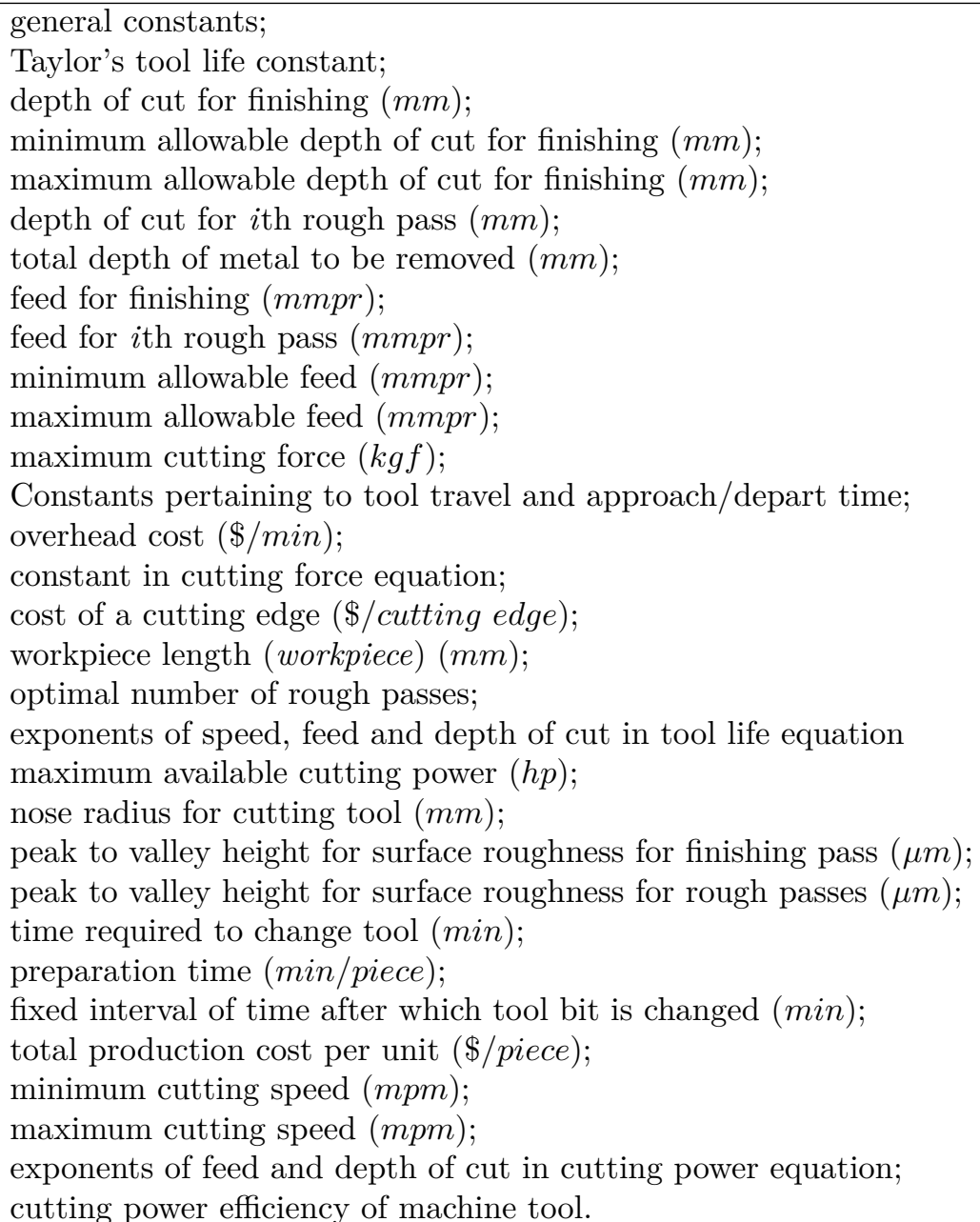 \\
\hline
\end{tabular}

Table 1: Notation of the manufacturing process data from Problem 1

(1) minimum speed

$$
\begin{aligned}
& f_{s}^{\left(\frac{q}{p}\right)} d_{s}^{\left(\frac{r}{p}\right)} \gtrsim \frac{C_{0}^{\left(\frac{1}{p}\right)}}{T_{p}^{\left(\frac{1}{p}\right)} v_{\max }} \\
& f_{r i}^{\left(\frac{q}{p}\right)} d_{r i}^{\left(\frac{r}{p}\right)} \gtrsim \frac{C_{0}^{\left(\frac{1}{p}\right)}}{T_{p}^{\left(\frac{1}{p}\right)} v_{\max }}
\end{aligned}
$$

(2) maximum speed

$$
\begin{aligned}
f_{s}^{\left(\frac{q}{p}\right)} d_{s}^{\left(\frac{r}{p}\right)} & \lesssim \frac{C_{0}^{\left(\frac{1}{p}\right)}}{T_{p}^{\left(\frac{1}{p}\right)} v_{\text {min }}} \\
f_{r i}^{\left(\frac{q}{p}\right)} d_{r i}^{\left(\frac{r}{p}\right)} & \lesssim \frac{C_{0}^{\left(\frac{1}{p}\right)}}{T_{p}^{\left(\frac{1}{p}\right)} v_{\text {min }}}
\end{aligned}
$$

(3) minimum feed and surface

$$
f_{s} \gtrsim f_{\min } \quad f_{r i} \gtrsim f_{\text {min }}
$$

(4) maximum feed and surface

$$
\begin{gathered}
f_{s} \lesssim \min \left(f_{\max }, \sqrt{8 R R_{\max }}\right) \\
f_{r i} \lesssim \min \left(f_{\max }, \sqrt{8 R R_{r}}\right)
\end{gathered}
$$

(5) cutting force

$$
k_{1} f_{s}^{\mu} d_{s}^{\mu} \lesssim F_{\max } \quad k_{1} f_{r i}^{\mu} d_{r i}^{\mu} \lesssim F_{\max }
$$

(6) cutting power

$$
\begin{aligned}
f_{s}^{\left(\mu-\frac{q}{p}\right)} d_{s}^{\left(\nu-\frac{r}{p}\right)} & \lesssim \frac{6120 \eta T_{p}^{\left(\frac{1}{p}\right)} P_{\max }}{k_{1} C_{0}^{\left(\frac{1}{p}\right)}} \\
f_{r i}^{\left(\mu-\frac{q}{p}\right)} d_{r i}^{\left(\nu-\frac{r}{p}\right)} & \lesssim \frac{6120 \eta T_{p}^{\left(\frac{1}{p}\right)} P_{\max }}{k_{1} C_{0}^{\left(\frac{1}{p}\right)}}
\end{aligned}
$$

(7) minimum depth of cut

$$
d_{s} \gtrsim d_{m i n} \quad d_{r i} \gtrsim d_{m i n}
$$

(8) maximum depth of cut

$$
d_{s} \lesssim d_{\max } \quad d_{r i} \lesssim d_{\max }
$$

(9) total depth

$$
d_{t}=d_{s}+\sum_{i=1}^{n o r} d_{r i}
$$

The term (1a) in the objective function is the total production cost for the finishing pass and the second is the total production cost for the rough passes. The set of constraints (1b) and (1c) ensures that the cutting speeds from finishing and roughing are within allowable range. The set of constraints (1d) and (1e) represents the feed rate constraints 
for both finishing pass and rough passes receptively. Force constraints are represented by the set of constraints (1f). The set of constraints (1g) represents the power constraints. The depth of cut for a finishing pass must be within the allowable range in the set of constraints (1h) and (1i). Finally, the constraint set $(1 \mathrm{j})$ ensures that the total subdivisions of depths of cut in finishing and rough passes are equal to the required depth of cut.

\section{Uncertainty in the set of constraints}

Normally, the lack of information the obtained data from real-world problems is represented by uncertainty, which will be dealt with fuzzy logic in this work. This lack of information can be found in the set of constraints, that is formulated by coefficients, decision variable, right-hand-side term, and/or order relation. In this work, the uncertain data is represented in the order relation.

Two approaches are developed to solve convex optimization problems under uncertain order relation in the set of constrains. The first approach is based on the linear fuzzy programming methods developed by Zimmerman, [13], where the decision maker must define an aspiration level to transform an original objection function into a constraint one. The second approach is based on the linear programming developed by Tanaka, Osada and Asai, [11], where an auxiliary function is defined in order to find satisfaction level between objective function and set of constraints.

\subsection{Extending Zimmerman's approach}

This approach transforms a fuzzy convex optimization problem into a classical convex optimization one by defining an aspiration level, which belongs to the objective function space and represents the expected value by decision maker.

A general convex optimization problem under uncertain order relation in the set of constraints can be written in the following way:

$$
\begin{array}{ll}
\widetilde{\min } & g_{0}(\mathbf{x}) \\
\text { s.t. } & g_{i}(\mathbf{x}) \lesssim b_{i}, \quad i=1, \ldots, m \\
& x \geq 0,
\end{array}
$$

where $\widetilde{\min }$ and $\lesssim$ represents the uncertain order relation. The decision maker can be define an aspiration level and then the objective function in Problem (2) can be transformed into a constraint function as

$$
g_{0}(\mathbf{x}) \lesssim b_{0}
$$

where $b_{0}$ is the aspiration level. From uncertain order relation, a linguistic variable for each constraint function, which describes a allowable violation, can be defined and the new set of constraints in the following way

$$
\begin{aligned}
& g_{j}(\mathbf{x}) \leq b_{j}+t_{j}, \quad j=0, \ldots, m \\
& \mathbf{x} \geq \mathbf{0} \\
& 0 \leq t_{j} \leq T_{j}
\end{aligned}
$$

where $t_{j}$ represents the allowable violation for the $j$-th constraint and $T_{j}$ represents the maximum allowable violation for the $j$-th constraint.

The allowable violation is described by the membership function $\mu_{j}\left(g_{j}(\mathbf{x})\right)$ as follows

$$
\mu_{j}\left(g_{j}(\mathbf{x})\right)= \begin{cases}0, & \text { se } t_{j}>T_{j} \\ 1-\frac{t_{j}}{T_{j}}, & \text { se } 0 \leq t_{j} \leq T_{j} \\ 1, & \text { se } t_{j}<0\end{cases}
$$

In order to aggregate all the membership function, a t-norm can be used as follows

$$
\begin{aligned}
& \bigwedge_{0 \leq j \leq m} \bigwedge_{\mathbf{x}} \mu_{j}\left(g_{j}(\mathbf{x})\right) \in[0,1] \\
& 0 \leq t_{j} \leq T_{j}
\end{aligned}
$$

where $\bigwedge$ is the intersection function.

After using the t-norm to aggregate all fuzzy sets, a satisfaction level can be obtained and the new objective is to maximize this satisfaction level for all constraints. With this in mind, this level is determine for each $j$ as follows

$$
S \leq 1-\frac{t_{j}}{T_{j}} \Leftrightarrow T_{j} S \leq T_{j}-t_{j} \Leftrightarrow T_{j} S+t_{j} \leq T_{j} .
$$

Thus, convex optimization problems under uncertain order relation in the set of constraints can be reformulated by a classical convex optimization problem in the following way

$$
\begin{array}{cc}
\max & S \\
\text { s.t. } & T_{j} S+t_{j} \leq T_{j} \\
& g_{j}(\mathbf{x})-t_{j} \leq b_{j} \\
& t_{j} \leq T_{j} \\
& S, \mathbf{x}, t_{j} \geq 0, \quad j=0, \ldots, m
\end{array}
$$

Note that the objective function (3a) and the constraints $(3 \mathrm{~b})$ and $(3 \mathrm{~d})$ are linear, while constraint (3c) is convex.

\subsection{Extending Tanaka's approach}

This approach solves convex optimization problems under uncertain order relation in the set of constraints in two steps. In the first one, an equivalent convex optimization problem is obtained, based on $[4,10]$, while the second step finds the best satisfaction level according to the set of solutions achieved in previous step. 
The equivalent classical convex optimization problem to (2) is obtained by inserting a new decision variable $t_{j}$ for each constraint as follows

$$
\begin{array}{ll}
\widetilde{\min } & g_{0}(\mathbf{x}) \\
\text { s.a } & g_{i}(\mathbf{x}) \leq b_{i}+t_{i}, \quad i=1, \ldots, m \\
& x \geq 0
\end{array}
$$

An aspiration level is not used in this approach but the new decisions variables belong to an interval $\left[0, T_{j}\right]$, for each $j$. This interval can be used to define a membership function, $\mu_{i}(\mathbf{x}): \mathbb{R}^{n} \rightarrow[0,1](\forall i=$ $1, \ldots, m)$, on the following way

$$
\mu_{i}(x)= \begin{cases}1, & g_{i}(\mathbf{x}) \leq b_{i} \\ \frac{\left(b_{i}+T_{i}\right)-g_{i}(\mathbf{x})}{T_{i}}, & b_{i}<g_{i}(\mathbf{x})<b_{i}+T_{i} \\ 0, & g_{i}(\mathbf{x}) \geq b_{i}+T_{i}\end{cases}
$$

After defining the membership functions of the constraints, an aggregation operator can be used to combine all membership functions of the constraints on the following way

$$
\mu_{C}(\mathbf{x})=\min _{i=1}^{m} \mu_{i}(\mathbf{x}), \forall x \in \mathbb{R}^{n}
$$

Now, the aggregated membership function can be used to represent whether a point is feasible or not, according to the $\alpha$-cut level. So, a parametric set of constraints is defined as follow

$$
C_{\alpha}=\left\{\mathbf{x} \mid \mathbf{x} \in \mathbb{R}^{n}, \mu_{C}(\mathbf{x}) \geq \alpha\right\}, \forall \alpha \in[0,1] .
$$

Thus, a convex optimization problem under uncertain order relation of the set of constraints can be transformed into a classical convex optimization problem, which depends on the parameter level $\alpha$, as follows

$$
\begin{array}{ll}
\widetilde{\min } & g_{0}(\mathbf{x}) \\
\text { s.a } & \mathbf{x} \in C_{\alpha}, \quad \alpha \in[0,1]
\end{array}
$$

For each $\alpha \in[0,1]$, Problem (8) can be solved by any classical optimization method and the solution reached belongs to a set of solutions. These solutions generate the fuzzy solution by using Representation Theorem. After this, the first step is concluded.

Once the fuzzy solution is computed the second step starts, which combines the membership functions of the set of constraints and the objective function, according to [3]. Hence,

$$
\mu_{D}=\mu_{C} \bigcap \mu_{G}
$$

where $\mu_{D}, \mu_{C}, \mu_{G}: \mathbb{R}^{n} \rightarrow[0,1]$.

The optimal decision in the feasible space is achieved with the membership function

$$
\mu_{D}\left(\mathbf{x}^{*}\right)=\max _{\mathbf{x} \in C_{\alpha^{*}}} \mu_{G}(\mathbf{x})
$$

where $C_{\alpha^{*}}$ is the optimal parametric set of constraints using $\alpha^{*}$-cut level

The fuzzy solution belongs to a bounded interval. In order to reach the limits of this interval, Problem (8) is solved for two ways: (i) set of constraints totally violated, $m$, and (ii) set of constraints totally satisfied, $M$.

$$
\begin{aligned}
& m=g_{0}\left(\mathbf{x}^{*}(0)\right)=\min _{\mathbf{x} \in C_{0}} g_{0}(\mathbf{x}) \\
& M=g_{0}\left(\mathbf{x}^{*}(1)\right)=\min _{\mathbf{x} \in C_{1}} g_{0}(\mathbf{x}),
\end{aligned}
$$

where $C_{0}$ and $C_{1}$ are the parametric sets of constraints for the $\alpha$-cut levels equal to 0 and 1 , respectively.

The values $m$ and $M$ are used to define the boundaries of the membership function $\mu_{G}(\mathbf{x})$ as follows

$$
\begin{aligned}
& \mu_{G}^{u}=1 \\
& \mu_{G}^{l}=\frac{m}{M} .
\end{aligned}
$$

According to the boundaries, the membership function of the objective function can be defined on the following way

$$
\mu_{G}(\mathbf{x})=\frac{m}{g_{0}(\mathbf{x})} .
$$

By replacing the membership function of the objective function (10) in Problem (9), we obtain

$$
\mu_{D}\left(\mathbf{x}^{*}\right)=\frac{1}{m} \min _{\mathbf{x} \in C_{\alpha^{*}}} g_{0}(\mathbf{x})
$$

In this moment, a classical optimization method can be used to reach the optimal solution subject to the feasible set of the parametric problem and the bounded interval of the membership function.

\section{Numerical experiments}

In order to illustrate the efficiency and robustness of two approaches that solve optimization problems under uncertain order relation in the set of constraints, the manufacturing process problem, which is formulated in Section 2, is solved by using them, described in Section 3.

The numerical results obtained by two proposed approaches are compared with the results in [1].

All the approaches are implemented in MATLAB ${ }^{\circledR}$ program by using the fmincon function in ToolBox Optimization package.

\subsection{Results and Analysis}

We use a mathematical formulation from manufacturing process problem, which was described in [8], to test the efficiency of the proposed approaches that optimization problem under fuzzy environment. In order to compare the results, the numerical data used in this work are given by [1] 


\begin{tabular}{|llll|}
\hline$D=50 \mathrm{~mm}$ & $p=5$ & $\zeta=1$ & $h_{1}=7 \times 10^{-4}$ \\
$d_{t}=60 \mathrm{~mm}$ & $q=1.75$ & $\mu=0.75$ & $h_{2}=0.3$ \\
$d_{\text {min }}=1.0 \mathrm{~mm}$ & $r=0.75$ & $\nu=0.95$ & $C_{0}=6 \times 10^{11}$ \\
$d_{\max }=3.0 \mathrm{~mm}$ & $k_{0}=\$ 0.5 / \mathrm{min}$ & $P_{\max }=5 \mathrm{~kW}$ & $F_{\max }=200 \mathrm{kgf}$ \\
$f_{\min }=0.1 \mathrm{~mm} / \mathrm{rev}$ & $k_{1}=108$ & $R=1.2 \mathrm{~mm}$ & $T_{\min }=25 \mathrm{~min}$ \\
$f_{\max }=0.9 \mathrm{~mm} / \mathrm{rev}$ & $k_{t}=\$ 2.5 /$ cutting edge & $R_{r}=100 \mu \mathrm{m}$ & $T_{\max }=45 \mathrm{~min}$ \\
$\nu_{\min }=5 \mathrm{~m} / \mathrm{min}$ & $t_{p}=0.75 \mathrm{~min} /$ piece & $R_{\max }=10 \mu \mathrm{m}$ & $T_{s}=25 \mathrm{~min}$ \\
$\nu_{\max }=500 \mathrm{~m} / \mathrm{min}$ & $t_{e}=1.5 \mathrm{~mm} /$ edge & $L=300 \mathrm{~mm}$ & \\
\hline
\end{tabular}

Table 2: Numerical data of the Problem (1)

\begin{tabular}{|c|c|c||c|c|c|}
\hline Constraints & \multicolumn{2}{|c||}{ Tolerances } & Constraints & \multicolumn{2}{c|}{ Tolerances } \\
\hline $1 \mathrm{~b}$ & $T_{1}$ & 4 & $1 \mathrm{f}$ & $T_{5}$ & 10 \\
$1 \mathrm{c}$ & $T_{2}$ & 200 & $1 \mathrm{~g}$ & $T_{6}$ & 4 \\
$1 \mathrm{~d}$ & $T_{3}$ & 0 & $1 \mathrm{~h}$ & $T_{7}$ & 0 \\
$1 \mathrm{e}$ & $T_{4}$ & 1 & $1 \mathrm{i}$ & $T_{8}$ & 0 \\
\hline
\end{tabular}

Table 3: Maximum levels of allowable tolerance

According to the proposals, a maximum tolerance for each constraint function can be allowed and this tolerances are described in Table 3.

Table 4 shows the obtained optimal solutions, for different values of the total depth of metal to be removed, $d_{t}$, to Problem (1) where the tolerances of all constraint functions are totally violated. Also, the iteration number that are necessary to converge up to each optimal solution is shown.

Table 5 shows the obtained solutions from AlAhmari's approach [1], which were reached by using the LINGO program. However, the iteration number and computational time are not informed. Thus, this approach is presented only to compare the optimal solution and its cost with the proposed approaches in this work.

The satisfaction levels achieved by all proposed approaches for each parameter $d_{t}$, total depth of metal to be removed are shown, in Table 6 . In this same table, the iteration number for each parameter is presented.

\begin{tabular}{|c|c|c|c|}
\hline Approach & $d_{t}$ & $\mu$ & iterations \\
\hline & 6 & $100 \%$ & 3 \\
& 8 & $100 \%$ & 5 \\
Classical & 8.5 & $100 \%$ & 5 \\
Approach & 9 & $100 \%$ & 3 \\
& 9.5 & $100 \%$ & 6 \\
& 10 & $100 \%$ & 6 \\
\hline \hline & 6 & $83.56 \%$ & 13 \\
Extending & 8 & $83.97 \%$ & 51 \\
Zimmermann's & 8.5 & $84.07 \%$ & 34 \\
Approach & 9 & $84.20 \%$ & 14 \\
& 9.5 & $84.23 \%$ & 56 \\
& 10 & $84.44 \%$ & 58 \\
\hline \hline & 6 & $81.34 \%$ & 14 \\
Extending & 8 & $82.08 \%$ & 43 \\
Tanaka's & 8.5 & $81.95 \%$ & 33 \\
Approach & 9 & $81.70 \%$ & 12 \\
& 9.5 & $82.70 \%$ & 56 \\
& 10 & $82.70 \%$ & 58 \\
\hline
\end{tabular}

Table 6: Satisfaction level of results to Problem (1).

It is clear to see that extending approaches need more iterations than the classical one and the satisfaction level for each solution is less than $100 \%$. However, the objective function value obtained for both extending approaches is better and the all satisfaction levels are more than $80 \%$. With this in mind, the decision maker has to do a tradeoff between objective function value and the satisfaction level. We highlight that the extending Tanaka's approach have two steps and the first step solves at least twice a parametric problem, as described in [4], in order to reach the minimum and maximum value with the allowable tolerance.

\section{Conclusion}

Convex Optimization problems are very important in a variety of both theoretic and practical areas. In this context this paper has proposed two extending approaches to solve convex optimization problem with fuzzy order relation in the set of constraints, which were developed to solve the linear case. The proposals have been validated through the problem for determining the machining parameters in multipass turning operations.

The first extending approach is based on the linear method developed by Zimmermann, which obtains an equivalent classical formulation from the fuzzy optimization problem by defining an aspiration level to transform an original objection function into a constraint function. The drawback of this approach is to define the aspiration level, which is chosen by the decision maker that is the expert in this problem.

The second extending approach is based on the linear method developed by Tanaka, Okuda, and Asai. The original approach make a tradeoff between the satisfaction level and the violation of the set of constraints. On the one hand, the decision maker has to define the interval in which the satisfaction level belongs. On the other hand, the interval boundaries are reached by solving an equivalent parametric problem, which is the case in this work and we has described as first step from this extending approach.

\section{Acknowledgments}

The authors want to thank the support provided by the Brazilian agencies CNPQ and CAPES. 


\begin{tabular}{|c|c|c|c|c|c|c|c|c|c|c|c|}
\hline Approach & $\begin{array}{c}d_{t} \\
(m m)\end{array}$ & $\begin{array}{c}f_{s} \\
(m m)\end{array}$ & $\begin{array}{c}f_{1} \\
(m m)\end{array}$ & $\begin{array}{c}f_{2} \\
(m m)\end{array}$ & $\begin{array}{c}f_{3} \\
(m m)\end{array}$ & $\begin{array}{c}d_{s} \\
(m m)\end{array}$ & $\begin{array}{c}d_{1} \\
(m m)\end{array}$ & $\begin{array}{c}d_{2} \\
(m m)\end{array}$ & $\begin{array}{c}d_{3} \\
(m m)\end{array}$ & $\begin{array}{c}U \\
(\$ / \text { piece })\end{array}$ & Iter. \\
\hline & 6 & 1.31 & 1.9 & - & - & 2.999 & 3.001 & - & - & 1.325 & 5 \\
\hline First Step & 8 & 1.31 & 1.9 & 1.9 & - & 1.999 & 3.000 & 3.001 & - & 1.759 & 28 \\
\hline Extending & 8.5 & 1.31 & 1.9 & 1.9 & - & 2.500 & 3.000 & 3.000 & - & 1.767 & 19 \\
\hline Tanaka's & 9 & 1.31 & 1.9 & 1.9 & - & 3.000 & 3.000 & 3.000 & - & 1.773 & 5 \\
\hline approach & 9.5 & 1.31 & 1.9 & 1.9 & 1.9 & 1.000 & 2.833 & 2.833 & 2.833 & 2.179 & 39 \\
\hline & 10 & 1.31 & 1.9 & 1.9 & 1.9 & 1.000 & 3.000 & 3.000 & 3.000 & 2.184 & 41 \\
\hline
\end{tabular}

Table 4: Results of Problem (1) where the constraint tolerances are totally violated.

\begin{tabular}{|c|c|c|c|c|c|c|c|c|c|c|c|}
\hline Approach & $\begin{array}{c}d_{t} \\
(\mathrm{~mm})\end{array}$ & $\begin{array}{c}f_{s} \\
(\mathrm{~mm})\end{array}$ & $\begin{array}{c}f_{1} \\
(\mathrm{~mm})\end{array}$ & $\begin{array}{c}f_{2} \\
(\mathrm{~mm})\end{array}$ & $\begin{array}{c}f_{3} \\
(\mathrm{~mm})\end{array}$ & $\begin{array}{c}d_{s} \\
(\mathrm{~mm})\end{array}$ & $\begin{array}{c}d_{1} \\
(\mathrm{~mm})\end{array}$ & $\begin{array}{c}d_{2} \\
(\mathrm{~mm})\end{array}$ & $\begin{array}{c}d_{3} \\
(\mathrm{~mm})\end{array}$ & nor & $\begin{array}{c}U \\
(\$ / \text { piece })\end{array}$ \\
\hline Al-Ahmari's & 6 & 0.310 & 0.566 & - & - & 3.000 & 3.000 & - & - & 1 & 1.939 \\
Approach & 8 & 0.310 & 0.900 & 0.585 & - & 3.000 & 2.079 & 2.921 & - & 2 & 2.481 \\
& 9 & 0.310 & 0.713 & 0.566 & - & 3.000 & 2.500 & 3.000 & - & 2 & 2.550 \\
& 9.5 & 0.310 & 0.566 & 0.566 & - & 3.000 & 3.000 & 3.000 & - & 2 & 2.619 \\
& 10 & 0.310 & 0.606 & 0.900 & 0.900 & 3.000 & 2.342 & 2.079 & 2.079 & 3 & 2.953 \\
& 6 & 0.310 & 0.566 & - & - & 3.000 & 3.000 & - & - & 1 & 1.939 \\
Classical & 8 & 0.310 & 0.713 & 0.712 & - & 3.000 & 2.500 & 2.500 & - & 2 & 2.481 \\
Approach & 8.5 & 0.310 & 0.632 & 0.632 & - & 3.000 & 2.750 & 2.750 & - & 2 & 2.550 \\
& 9 & 0.310 & 0.566 & 0.566 & - & 3.000 & 3.000 & 3.000 & - & 2 & 2.619 \\
& 9.5 & 0.310 & 0.854 & 0.854 & 0.854 & 3.000 & 2.167 & 2.167 & 2.167 & 3 & 2.953 \\
\hline & 10 & 0.310 & 0.777 & 0.777 & 0.777 & 3.000 & 2.334 & 2.334 & 2.332 & 3 & 3.022 \\
\hline & 6 & 0.474 & 1.0 .64 & - & - & 3.000 & 3.000 & - & - & 1 & 1.644 \\
Zimtending & 8 & 0.470 & 1.060 & 1.060 & - & 2.000 & 3.000 & 3.000 & - & 2 & 2.156 \\
Approach & 8.5 & 0.469 & 1.060 & 1.060 & - & 2.500 & 3.000 & 3.000 & - & 2 & 2.173 \\
& 9 & 0.468 & 1.058 & 1.058 & - & 3.000 & 3.000 & 3.000 & - & 2 & 2.187 \\
& 9.5 & 0.468 & 1.058 & 1.058 & 1.058 & 1.000 & 2.833 & 2.833 & 2.833 & 3 & 2.645 \\
\hline \hline & 10 & 0.466 & 1.056 & 1.056 & 1.056 & 1.000 & 3.000 & 3.000 & 3.000 & 3 & 2.654 \\
\hline Extending & 6 & 0.493 & 1.083 & - & - & 3.000 & 3.000 & - & - & 1 & 1.629 \\
Tanaka's & 8 & 0.485 & 1.075 & 1.075 & - & 2.000 & 3.000 & 3.000 & - & 2 & 2.143 \\
Approach & 8.5 & 0.487 & 1.077 & 1.077 & - & 2.500 & 3.000 & 3.000 & - & 2 & 2.156 \\
& 9 & 0.485 & 1.075 & 1.075 & - & 3.000 & 3.000 & 3.000 & - & 2 & 2.170 \\
& 9.5 & 0.477 & 1.067 & 1.067 & 1.067 & 1.000 & 2.833 & 2.833 & 2.833 & 3 & 2.635 \\
& 10 & 0.478 & 1.068 & 1.068 & 1.068 & 1.000 & 3.000 & 3.000 & 3.000 & 3 & 2.641 \\
\hline
\end{tabular}

Table 5: Results of Problem (1) for all approaches.

\section{References}

[1] A.M.A. Al-Ahmari. Mathematical model for determining machining parameters in multpass turning operations with constraints. International Journal Production Research, 39 (15), 3397-3376, 2001.

[2] S. Bharathi Raja and N. Baskar. Optimization techniques for machining operations: a retrospective research based on various mathematical models. International Journal of Advanced Manufacturing Technology, 48, 1075-1090, 2010.

[3] R. E. Bellman and L. A, Zadeh. Decisionmarking in a fuzzy environment. Management Science, 17 (4), B141-B164, 1970.

[4] C. Cruz, R. C. Silva, and J. L. Verdegay. Extending and relating different approaches for solving fuzzy quadratic problems. Fuzzy Optimization and Decision Making, 10(3), 193-210, 2011.

[5] C. Cruz, R. C. Silva, J. L. Verdegay and A. Yamakami. A survey of fuzzy quadratic programming. Recent Patents on Computer Science, 1(3), 182-193, 2008.

[6] I. Mukherjee and P. K. Ray. A review of optimization techniques in metal cutting processes. Computers \& Industrial Engineering, 50, 15-34,
2006.

[7] C. V. Negoita, D. A. Ralescu. Applications of fuzzy sets to systems analysis, Birkhauser Verlag, Stuttgard, 1975.

[8] Y. Shin and Y. Joo. Optimization of machining conditions with practical constraints. International Journal of Production Research, 30, 2907 2919, 1992.

[9] R. C. Silva, C. Cruz, J. L. Verdegay and A. Yamakami. A survey of fuzzy convex programming models. In W. LODWICK and J. KACPRZYK (eds), Fuzzy Optimization, Studies in Fuzziness and Soft Computing, vol. 254, 127-143, Springer, 2010.

[10] J. L. Verdegay. Fuzzy mathematical programming, in: M. M. GUPTA, E. SANCHES (eds.), Fuzzy Information and Decision Procsses, North-Holland Publishing Company, Amsterdam, 1982.

[11] H. Tanaka, T. Okuda, K. Asai. On fuzzymathematical programming, Journal of Cybernetics, 3 (4), 37?46, 1974.

[12] L. A. Zadeh. Fuzzy Sets. Information and Control, 8, 338-353, 1965.

[13] H. J. Zimmermann. Fuzzy Mathematical Programming. Computer \& Operation Research, 10 (4), 291-298, 1983. 